\title{
TCD neurosonology: A window to view thinking
}

\author{
DIANNE M. O'DELL and A. E. ROBERTS \\ Catawba College, Salisbury, North Carolina \\ and \\ WILLIAM M. MCKINNEY \\ The Bowman Gray School of Medicine, Winston-Salem, North Carolina
}

\begin{abstract}
Bilateral transcranial Doppler ultrasound measures of blood flow velocities in the middle cerebral artery (MCA) were taken from college students during three 45-sec cognitive activities. Each activity (remembering letters, decision making, and solving problems) was preceded by a 60 -sec baseline period and involved three different 15 -sec tasks. MCA blood flow velocities increased significantly over baseline throughout the letter recall and problem-solving tasks but not during decision making. Within-task indices were biphasic; initially, velocities were elevated markedly, but then they lessened. Velocities were maintained above baseline by the letter recall and problemsolving tasks, but they diminished to match baseline in the final portions of the decision-making task. Velocity differences due to the hemisphere insonated (right or left) were not reliable.
\end{abstract}

Transcranial Doppler (TCD) neuroimaging is a noninvasive procedure that relies on ultrasound signals to measure blood flow velocity in the intracranial arteries. These arteries are readily insonated through a "transtemporal window" by a transducer positioned on the scalp, and each artery provides discernible measurement characteristics that facilitate identification (see Aaslid, 1986). Blood flow velocities (in centimeters/second) in the intracranial arteries in normal subjects show only minor between-hemisphere differences and relatively small between-session differences (Sorteberg, Langmoen, Lindegaard, \& Nornes, 1990). Velocities are not influenced by subject gender or handedness but are lower in older subjects (Droste, Harders, \& Rastogi, 1989a, 1989b). Typically, the velocity measures from the middle cerebral artery (MCA) are higher than those from the anterior cerebral artery (ACA), which, in turn, are higher than those from the posterior cerebral artery (Sorteberg et al., 1990). One important feature of TCD is that changes in hemovelocities are open to immediate and continuous monitoring. In contrast, other cerebral blood flow measurement techniques (e.g., positron emission tomography, PET) rely on post-perfusion assessments.

TCD has primarily been used for neurological diagnosis and screenings (Aaslid, 1986; Harders, 1986). Recently in Germany, Droste and his associates (Droste et al., 1989a, 1989b) extended the use of TCD into the realm of nonmedical assessment. In a series of related experiments, bilateral changes in blood flow velocities in the MCA were monitored during sets of cognitive activities.

Droste et al. (1989a) recorded blood flow velocities in the left and right MCAs during six cognitive tasks: reading aloud, dot/distance estimation, noun finding, spatial per-

D. M. O'Dell now is at Virginia Commonwealth University, Richmond, VA. All correspondence may be addressed to A. E. Roberts, Department of Psychology, Catawba College, Salisbury, NC 28144. ception, multiplication, and face recognition. The six 90 sec tasks alternated with baseline periods of $42 \mathrm{sec}$ (e.g., baseline-task-baseline-task). Bilateral increases in velocity occurred during all tasks, relative to an average baseline value (the last $9 \mathrm{sec}$ of the six immediately preceding baseline periods). Velocity increases ranged from $1.6 \%$ to $10.6 \%$ (across tasks) and were taken as evidence of the cortical activity induced by the cognitive tasks. A betweenhemisphere habituation effect also was found; that is, velocities were reduced in the side that was insonated last.

Droste et al. (1989b, Experiment 1) followed the same basic procedures as they did in the 1989 a study to measure MCA blood flow velocities during silent and spoken performances of three cognitive tasks: reading abstract four-syllable nouns, finding nouns of a given letter from among a list of nouns, and multiplication. Blood flow velocities during each task increased bilaterally over baseline. However, the requirement of spoken performance in the reading task induced significantly higher velocities than did the requirement of silent performance; no reliable differences between response requirements were found for the other two tasks.

Harders, Laborde, Droste, and Rastogi (1989, Experiment 1) insonated the MCA during three presumed righthemisphere tasks (e.g., spatial imagination) performed silently, and three presumed left-hemisphere tasks (e.g., finding nouns) performed aloud. MCA velocities in the right-hemisphere tasks were higher during right-side insonations, in comparison with left-side measures. Reliable side differences in measures were not obtained in lefthemisphere tasks.

O'Dell, Roberts, and McKinney (in press) insonated the MCA during three 90 -sec memory tasks: remembering a set of letters, generating and maintaining images of personal experiences, and answering general knowledge questions. These tasks induced bilateral increases in blood 
flow velocities, but differences attributable to the kind of task, the side insonated (right or left), or the gender of subjects were not found.

The purpose of the present experiment was to use TCD to monitor bilateral changes in MCA hemovelocity during three cognitive tasks selected to represent different dimensions of thinking: remembering letters, solving problems, and making decisions. The letter recall task served as a partial replication of the O'Dell et al. (in press) study. The latter two tasks were viewed as being less memorybound than the former, with the primary difference between the two centering on the response product. Problem solving typically leads to an outcome that can be judged as correct or incorrect. The outcome to a decisionmaking task is more open ended and not judged along a correct-incorrect dimension (see Matlin, 1989).

\section{METHOD}

\section{Subjects}

After giving their informed consent, 6 males and 9 females served as subjects. All subjects were college students (mean age: 18.9 years) who reported no medically diagnosed problems and were not taking medication, and all but 3 were right-handed.

\section{Apparatus}

A TC2-64B transcranial Doppler (EME GmbH, Überlingen, Germany) was used to measure blood flow velocities. The MCA/ACA bifurcation served as a reference point for locating the MCA. The MCA was insonated distal to the bifurcation at a depth of 50-55 mm with an ultrasound intensity of $100 \mathrm{~mW} / \mathrm{cm}^{2}$. The transducer was secured in a head ribbon (EME: IMP2L monitoring probe) to permit continuous measurement. Timeaveraged mean blood flow velocity values were provided by the machine in centimeter values every $3 \mathrm{sec}$. The velocity values were routed to a 19-in. TV monitor (located in back of the subject) to magnify display and facilitate hand recording of the values. After insonation was accomplished, audio signals from the TCD unit and the TV were turned off.

\section{Procedure}

The experiment followed a $2 \times 6$ factorial design with repeated measures on both factors. Insonation side (right and left) served as one factor. The six experimental phases made up the second factor; subjects performed three 45-sec cognitive tasks, each of which was preceded by a 60 -sec rest period. The tasks were the following: remembering sets of three letters, making decisons based on scenarios, and solving problems.

Each subject was tested individually while seated comfortably in a reclining chair in a dimly lit, sound-attenuated room. After the MCA was insonated, a 5-min rest period began, during which the subject remained sitting with the eyes closed. The final $30 \mathrm{sec}$ of this rest period provided baseline measures for the first cognitive phase.
The letter-remembering phase was based on the Peterson and Peterson (1959) procedure. The subject was read a set of three letters followed by a two-digit number, silently added or subtracted, as instructed, by threes or fours for $15 \mathrm{sec}$, and then recalled the three letters in the order given. Three different sets of letters were given in this phase. In the decisionmaking phase, the subject was given scenarios such as the following:

Assume that you are "just getting by" academically and must choose between two majors of equal interest to you. Major $A$ is very difficult but is guaranteed to lead to a job after graduation. Major B is easy but may not lead to a job after graduation. Which major would you choose, and why?

After $15 \mathrm{sec}$, the subject was asked to respond. Three different scenarios were provided during this phase. The procedures in the problem-solving phase were identical to those for decision making, except that a specific answer could be given to the questions-for example, "Four men shook hands with each other just once. How many handshakes were made?" Three different problems were given in this phase.

Half the subjects began the experiment under right-side insonation and half began under left-side insonation. The six phases were given twice-once under each insonation. The sequences of the phases were counterbalanced for each side. Similar, but not identical, tasks were given during the second insonation.

Subjects' verbalizations, movements, and vision during measurements were minimized so that increases in blood flow velocities represented self-generated brain activity rather than reactions to extraneous environmental stimuli (Roland \& Friberg, 1985). No TCD measurements were taken during the instructions or while the subjects answered at the end of a task. Questions from the subjects were not permitted during task periods. The subjects kept their eyes closed throughout the experiment.

Separate mean velocities were calculated for each subject from each of the six phases for statistical analysis. The final $30 \mathrm{sec}$ of each rest period (10 values) were used to obtain a single mean for that baseline. Measures were taken only during a target cognitive activity, so a single mean was obtained from the entire $45 \mathrm{sec}$ of each phase (15 values).

\section{RESULTS}

The group mean velocities from the six phases are presented for both the right- and left-side insonations in Table 1 . The standard deviations given in the table are indices of variability that typically accompany group means. The $S D_{w}$ values are indices of the consistency of the means obtained for each subject; they are averaged within-subject $S D$ values.

A $2 \times 6$ factorial analysis of variance (with repeated measures on both factors) was used to analyze the velocity data $(\alpha=.05)$. The velocity differences between rightand left-side insonations $(M=57.0$ and 57.8 , respectively) and the side $\times$ task interaction were not reliable $(F \mathrm{~s}<1)$. However, the differences between the six phases

Table 1

Mean Blood Flow Velocities (in Centimeters/Second) Obtained During the Six Phases of the Experiment

\begin{tabular}{|c|c|c|c|c|c|c|c|c|}
\hline \multirow[b]{2}{*}{ Phases } & \multicolumn{3}{|c|}{ Right-Side Insonation } & \multicolumn{3}{|c|}{ Left-Side Insonation } & \multicolumn{2}{|c|}{ Combined } \\
\hline & $\boldsymbol{M}$ & $S D$ & $S D_{\mathrm{w}}$ & $\boldsymbol{M}$ & $S D$ & $S D_{w}$ & $\boldsymbol{M}$ & $S D$ \\
\hline Baseline & 56.2 & 7.66 & 2.32 & 57.3 & 7.11 & 2.75 & 56.7 & 6.35 \\
\hline Letter recall & 58.4 & 7.67 & 2.59 & 59.7 & 8.56 & 3.00 & 59.0 & 6.90 \\
\hline Baseline & 56.3 & 7.14 & 2.26 & 56.9 & 7.05 & 2.86 & 56.6 & 5.89 \\
\hline Decision making & 57.4 & 7.54 & 3.42 & 58.1 & 7.66 & 3.22 & 57.8 & 6.21 \\
\hline Baseline & 56.4 & 7.49 & 2.49 & 56.0 & 7.35 & 2.61 & 56.2 & 6.66 \\
\hline Problem solving & 57.5 & 8.35 & 2.83 & 58.9 & 7.66 & 2.83 & 58.2 & 6.96 \\
\hline
\end{tabular}

Note- $S D$, between-subject standard deviation values associated with each mean; $S D_{w}$, indices of within-subject variability-that is, averages of $S D$ values obtained from each subject during a phase. 
were significant $\left[F(5,70)=6.45, M S_{e}=5.48 ; \eta^{2}=31 \%\right]$. Bilaterally averaged velocity values for each subject from each phase (given in Table 1 as "combined" values) were analyzed with Newman-Keuls tests. Flow velocities obtained during the letter recall and problem-solving tasks were elevated significantly over their respective baselines, but the velocity difference between decision making and its baseline was unreliable. Finally, differences in velocities between the three baseline periods were not significant.

Figure 1 presents the group mean velocities during the three cognitive tasks and their respective baselines. Figure 1 shows that mean velocities during each of the sets of probes within the cognitive tasks were elevated initially, but the degree of increase appears to lessen over the 15 -sec probe.

To test for this possibility, the six values from each of the five 3-sec probe intervals of a phase were averaged for each subject. These five averages, along with the bilaterally averaged baseline value, were analyzed with a $3 \times 6$ factorial analysis of variance (with repeated measures on both factors). The three cognitive tasks served as the other factor. These averages are given in Figure 2 in terms of the degree of change in blood flow velocity from baseline in each of the five probe intervals (this arrangement gives a common point of reference for the tasks). Pertinent to this analysis were the significant changes between measurement intervals $\left[F(5,70)=24.18, M S_{\mathrm{e}}=2.80\right]$ and the significant task $X$ interval interaction $[F(10,140)$ $\left.=2.05, M S_{\mathrm{e}}=1.60\right]$. Newman-Keuls tests showed that blood flow velocities were elevated over baseline in the first two task intervals, and the degree of elevation did not differ between the three tasks. Velocities in the final two intervals of letter recall and problem solving were less than those in the first two intervals but remained above

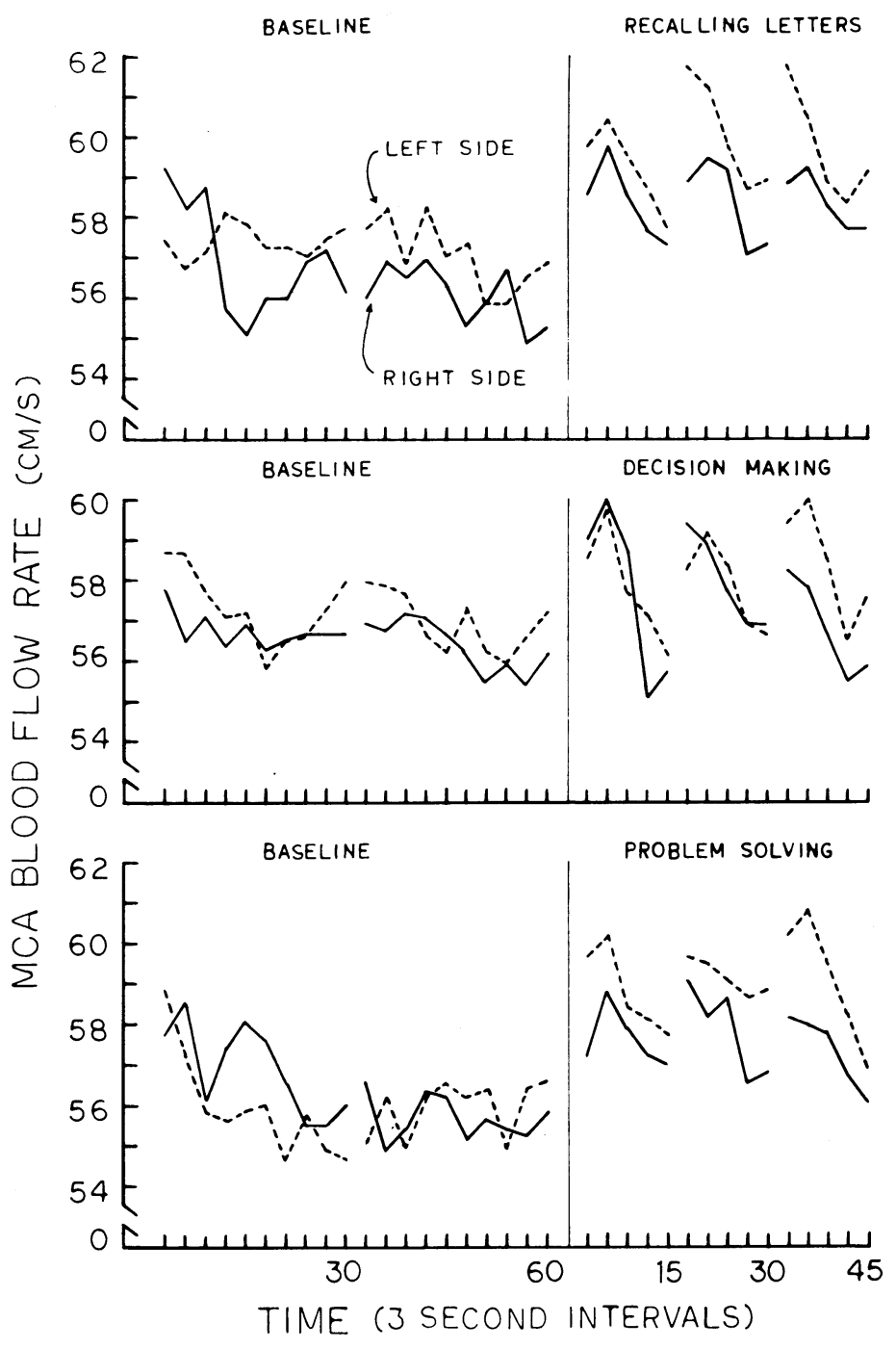

Figure 1. Mean medial cerebral artery (MCA) flow rates (in centimeters/ second) obtained during each probe in each cognitive task and from the baseline period immediately preceding each task. Values to the right of the break in the baseline slopes were used in the statistical analyses. 


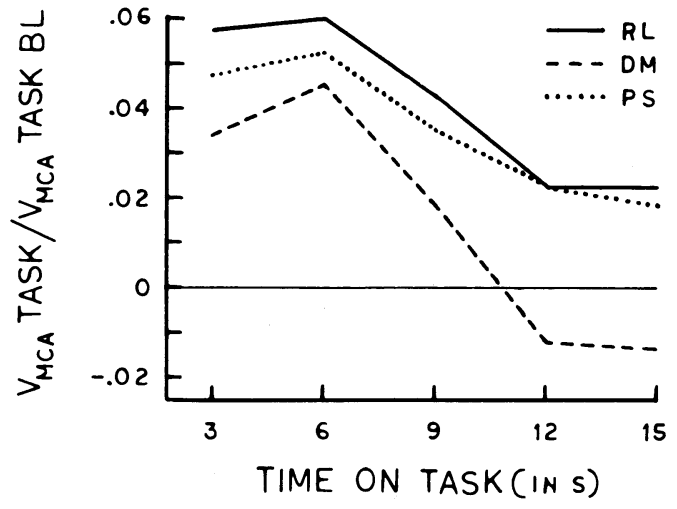

Figure 2. Changes in bilaterally averaged blood flow velocities during recalling-tetter (RL), decision-making (DM), and problem-solving (PS) probes. The changes are given relative to the baseline of each task (the horizontal 0-line).

baseline. However, blood flow velocities in the final two intervals of decision making were comparable to baseline.

\section{DISCUSSION}

In the present experiment, TCD was used to monitor bilateral changes in MCA hemovelocity during three cognitive activities. Our subjects, like those in other studies, showed changes in blood flow velocities coincident with their performance of a cognitive task. Letter recall and problem solving induced an elevation in blood flow velocities throughout the duration of the tasks; the result from the letter recall task replicates the report by $O$ 'Dell et al. (in press). Moreover, these task-induced increases originated from the cortical activities prescribed by the cognitive task, since ancillary contributors (e.g., subject verbalizations, movements, and vision) were restricted. The degree of increase in velocity during these tasks was not bilaterally differentiated, which is consistent with other reports (Droste et al., 1989a, 1989b; O'Dell et al., in press).

Although the short-term remembering of letters and problem solving produced overall elevations in blood flow velocities, decision making did not. This outcome does not mean that hemovelocity was unaffected during decision making. Our within-task analysis showed the presence of a biphasic lineament in the elevation in hemovelocity; the degree in elevation present early in a task interval subsequently lessened. The letter recall and the problem-solving activities sustained the elevation over the later intervals, whereas the decision-making activity did not.

These biphasic patterns point to two issues of interest: the sources of the initial elevation, and the cognitive processing features that maintain the elevation. With respect to the first issue, the transitory elevation in velocity at the outset of a cognitive task is not unique to this study (Droste et al., 1989a, 1989b; Harders et al., 1989; O'Dell et al., in press). This elevation could be due to transitory respiratory or cardiovascular involvements (e.g., hypoventilation or blood pressure changes, respectively). ${ }^{1}$ But this peak could also follow from collateral cognitive activities of the subject, such as residual influences of listening to the instructions and/or heightened attentiveness to the verbal presentations of the probes. In the case of cognitive sources, we noted earlier that subject verbalizations accentuate the increase in blood flow velocity over those obtained during silent activities (Droste et al., 1989b, Experiment 1). Cognitive demands beyond those arising from the target task may similarly affect hemovelocities and contribute additively to the hemovelocity measures. In fact, PET analyses are often grounded on the occurrence of the additivity in effect to localize cognitive functions (see Posner, Petersen, Fox, \& Raichle, 1988). The effects of collateral sources on directly measured hemovelocity can be addressed in future research by adding and then removing potential sources during the measurement period.

With respect to the second issue, the maintenance of the elevation in blood flow velocity beyond the initial peak reflects the cognitive ac- tivities demanded by the task and generated by the subject. The procedure for the recall of letters required the subject to count backward during the measurement interval, and this activity maintained the elevation. The problem-solving questions similarly involved private manipulations during the measurement period, although they were not specifically prescribed-as, for example, those that were needed to determine a correct response. In contrast, the private activities necessary to produce a "decision" in response to the scenarios were less structured. Our subjects probably came to their decisions quickly, with little subsequent cogitation over the remainder of the interval; blood flow velocity then returned to baseline, coincidentally with the suspension of their cognitive processing. If so, directing the subjects toward more structured and extended decision-making strategies (e.g., "dimension comparisons"; see Halpern, 1989) should obviate this reduction.

Returning to the main focus of the experiment, TCD documented the increase in MCA hemovelocity induced by thinking tasks. Our report of changes in regional cerebral blood flow velocities during a cognitive activity is not new to the literature (see Lassen, Ingvar, \& Skinhøj, 1978). However, our study shows that modulations in hemovelocity occur within the period ascribed to a cognitive activity. TCD provides measures concomitant with the temporal dynamics of the task period (e.g., onset, duration, and offset), so changes in hemovelocity can be assessed immediately and conveniently. Moreover, TCD can capture these modulations to short task durations (15 sec in this experiment) as well as to more extended task durations (e.g., $90 \mathrm{sec}$ in Droste et al., 1989a, 1989b). This opportunity marks an advantage for TCD over its postperfusion (i.e., "after-the-fact") counterparts.

\section{REFERENCES}

AAsLID, R. (Ed.) (1986). Transcranial Doppler sonography. New York: Springer-Verlag.

Droste, D. W., Harders, A. G., \& RAstogi, E. (1989a). A transcranial Doppler study of blood flow velocity in the middle cerebral arteries performed at rest and during mental activities. Stroke, 20, 1005-1011.

Droste, D. W., Harders, A. G., R Rastogi, E. (1989b). Two transcranial studies on blood flow velocity in both middle cerebral arteries during rest and the performance of cognitive tasks. Neuropsychologia, 27, 1221-1230.

HARDERS, A. G. (1986). Neurosurgical applications of transcranial Doppler sonography. New York: Springer-Verlag.

Harders, A. G., LABorde, G., Droste, D. W., \& RAstogi, E. (1989). Brain activity and blood flow velocity changes: A transcranial Doppler study. International Journal of Neuroscience, 47, 91-102.

HALPERN, D. F. (1989). Thought and knowledge: An introduction to critical thinking (2nd ed.). Hillsdale, NJ: Erlbaum.

Lassen, N. A., IngVar, D. H., SkinhфJ, E. (1978). Brain function and blood flow. Scientific American, 239, 62-71.

Matun, M. W. (1989). Cognition (2nd ed.). New York: Holt, Rinehart \& Winston.

O'Dell, D. M., Roberts, A. E., McKinney, W. M. (in press). Transcranial sonographic monitoring of cognitive activities. Journal of Neuroimaging.

Peterson, L. R., \& Peterson, M. J. (1959). Short-term retention of individual items. Journal of Experimental Psychology, 58, 193-198.

Posner, M. I., Petersen, S. E., Fox, P. T., Raichle, M. E. (1988). Localization of cognitive operations in the human brain. Science, 240 , 1627-1631.

Roland, P. E., \& Friberg, L. (1985). Localization of cortical areas activated by thinking. Journal of Neurophysiology, 53, 1219-1243.

SORTEberg, W., LANGMOEN, I. A., LiNDEGAARD, K. F., \& NorNes, H. (1990). Side-to-side differences and day-to-day variations of transcranial Doppler parameters in normal subjects. Jourmal of Ultrasound in Medicine, 9, 403-409.

\section{NOTE}

1. R.W. Droste (personal communication, 1991) reminded us of these two possibilities.

(Manuscript received January 21, 1992.) 